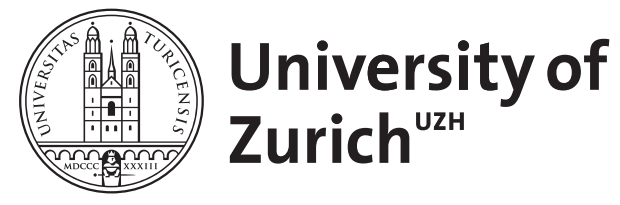
Archive

University of Zurich

University Library

Strickhofstrasse 39

CH-8057 Zurich

www.zora.uzh.ch

Year: 2018

\title{
Localisation of DBS Electrodes Post-Implantation, to CT or MRI? Which Is
} the Best Option?

\author{
Ellenbogen, Jonathan R ; Tuura, Ruth ; Ashkan, Keyoumars
}

DOI: https://doi.org/10.1159/000493576

Posted at the Zurich Open Repository and Archive, University of Zurich ZORA URL: https://doi.org/10.5167/uzh-157785

Journal Article

Published Version

Originally published at:

Ellenbogen, Jonathan R; Tuura, Ruth; Ashkan, Keyoumars (2018). Localisation of DBS Electrodes Post-Implantation, to CT or MRI? Which Is the Best Option? Stereotactic and functional neurosurgery, 96:347-348.

DOI: https://doi.org/10.1159/000493576 


\section{Letter to the Editor}

\section{Stereotactic and Functional Neurosurgery}

\section{Stereotact Funct Neurosurg 2018;96:347-348 \\ DOI: $10.1159 / 000493576$ \\ Localisation of DBS Electrodes Post-Implantation, to CT or MRI? Which Is the Best Option?}

\author{
Jonathan R. Ellenbogen ${ }^{\text {a }}$ Ruth Tuura ${ }^{\mathrm{b}}$ Keyoumars Ashkan ${ }^{\mathrm{c}}$ \\ ${ }^{a}$ Alder Hey Children's Hospital, Department of Neurosurgery, \\ Liverpool, UK; ${ }^{b}$ Center for MR Research, University Children's \\ Hospital, Zurich, Switzerland; ' Kings College Hospital, \\ Department of Neurosurgery, London, UK
}

Precise deep brain stimulator (DBS) lead and contact positioning is crucial for the therapeutic success of the procedure. Postoperative localisation imaging is therefore essential to verify the electrode position and avoid the need for additional repositioning surgery at a later date. Verification may be done by image fusion of the pre-operative stereotactic magnetic resonance imaging (MRI) with a post-implantation stereotactic computed tomography (CT) image or, alternatively, by stereotactic post-operative MRI. There is a debate on the choice of the best modality. The advantages of stereotactic MRI/CT fusion include direct visualisation of the metallic electrode contact with high-resolution CT, fast acquisition time of CT important for the comfort of awake patients and the safety of those undergoing surgery with a general anaesthetic, and the reduced costs of CT compared to MRI. This is in contrast to the inherent safety concerns of MRI in the presence of DBS electrodes, and the artefact limitations of a large eccentric signal void around the contacts which is seen on MRI as the contact itself cannot be directly visualised [1]. The main advantage of postimplantation stereotactic MRI is bypassing the need for post-implantation image fusion with the associated errors that fusion algorithms can introduce [2]. In recent years, however, continual improvements in CT/MRI fusion algorithms for the localisation of electrode placement have enabled post-implantation CT fused with pre-operative MRI to offer an accurate, reliable, and safe modality for assessing the anatomic location of DBS electrodes and active contacts [3-6].

Several recent papers have supported the use of post-implantation stereotactic MRI in DBS lead verification, addressing both the geometry of the electrode artefact and the safety concerns [7-9]. Hyam et al. [7] suggested that the lead hypointensity seen on postimplantation stereotactic MRI is an accurate representation of its real location within deep brain structures. However, in their paper, they assumed zero positioning error on the $z$-axis on post-operative MRI when comparing this to the literature values for the fusion accuracy, and this assumption is open to question. The electrodes cause through-plane as well as in-plane dephasing, resulting in a signal void which will extend past the tip of the electrode. Therefore, since the positioning error in this paper was only measured in-plane, a fairer comparison would be to compare this to the inplane fusion error. For neuroinspire ${ }^{\mathrm{TM}}$ fusion software, for example, this error would be $0.4 \mathrm{~mm}$, which is less than the $0.7 \mathrm{~mm}$ distance quoted with the post-implantation MRI technique $[6,7]$. Thus, the localisation error in using post-operative MRI, while small, is still greater than the fusion error achievable with the current state-of-the-art software packages due to the large variable electrode artefact seen on MRI, somewhere within which the actual contact lies.

With respect to MRI safety and DBS, some manufacturers now have DBS systems (e.g., Medtronic Activa and Boston Scientific Vercise Gevia) which are deemed safe for post-implantation MRI at 1.5-T under certain specific conditions. Clearly, the experience of the MRI physicist and the neuroradiologist is critical in implementing these conditions correctly to make the scan safe, an important consideration as such expertise may not be readily available at every DBS centre. Similarly, although 3-T MRI has been demonstrated to be safe in certain units in patients with implanted DBS systems, the results reported to date do not necessarily generalise to other scanners with different radiofrequency coil designs, and there are still ongoing concerns regarding its safety with no DBS manufacturer recommending this presently $[8,10]$.

Stereotactic localisation of DBS electrodes is an inherent component of the surgical process. The debate on the best means of achieving this is likely to continue. Post-implantation MRI and stereotactic post-operative CT fused with stereotactic pre-operative MRI both offer viable alternatives for post-operative verification of the electrode position, with an in-plane accuracy of within $1 \mathrm{~mm}$. However, in our view, stereotactic CT fused with pre-operative stereotactic MRI offers advantages in terms of a safer, faster, and less expensive examination, with an accuracy that is arguably comparable to or even slightly better than that of post-implantation stereotactic MRI, when state-of-the-art fusion algorithms are applied.

\section{Disclosure Statement \\ The authors have no conflicts of interest to declare.}

Funding Sources

There are no funding sources.

\section{References}

1 Pinsker MO, Herzog J, Falk D, Volkmann J, Deuschl G, Mehdorn M. Accuracy and distortion of deep brain stimulation electrodes on postoperative MRI and CT. Zentralbl Neurochir. 2008 Aug;69(3):144-7.

2 O'Gorman RL, Jarosz JM, Samuel M, Clough C, Selway RP, Ashkan K. $\mathrm{CT} / \mathrm{MR}$ image fusion in the postoperative assessment of electrodes implanted for deep brain stimulation. Stereotact Funct Neurosurg. 2009; 87(4):205-10.

\section{KARGER}

(C) 2018 S. Karger AG, Basel

E-Mail karger@karger.com

www.karger.com/sfn
Jonathan Ellenbogen

Department of Neurosurgery, Alder Hey Children's NHS Foundation Trust Eaton Road

Liverpool, Merseyside, L12 2AP (UK)

E-Mail jellenbogen@nhs.net 
3 Thani NB, Bala A, Swann GB, Lind CR. Accuracy of postoperative computed tomography and magnetic resonance image fusion for assessing deep brain stimulation electrodes. Neurosurgery. 2011 Jul;69(1):207-14.

4 Bot M, van den Munckhof P, Bakay R, Stebbins G, Verhagen Metman L. Accuracy of Intraoperative Computed Tomography during Deep Brain Stimulation Procedures: Comparison with Postoperative Magnetic Resonance Imaging. Stereotact Funct Neurosurg. 2017;95(3):183-8.

5 Mirzadeh Z, Chapple K, Lambert M, Dhall R, Ponce FA. Validation of CT-MRI fusion for intraoperative assessment of stereotactic accuracy in DBS surgery. Mov Disord. 2014 Dec;29(14):1788-95.

6 Geevarghese R, O'Gorman Tuura R, Lumsden DE, Samuel M, Ashkan K. Registration Accuracy of CT/MRI Fusion for Localisation of Deep Brain Stimulation Electrode Position: An Imaging Study and Systematic Review. Stereotact Funct Neurosurg. 2016;94(3):159-63.
7 Hyam JA, Akram H, Foltynie T, Limousin P, Hariz M, Zrinzo L. What you see is what you get: lead location within deep brain structures is accurately depicted by stereotactic magnetic resonance imaging. Neurosurgery. 2015 Sep;11(0 Suppl 3):412-9.

8 Sammartino F, Krishna V, Sankar T, Fisico J, Kalia SK, Hodaie M, et al. 3-Tesla MRI in patients with fully implanted deep brain stimulation devices: a preliminary study in 10 patients. J Neurosurg. 2017 Oct;127(4): 892-8.

9 Aviles-Olmos I, Kefalopoulou Z, Tripoliti E, Candelario J, Akram H, Martinez-Torres I, et al. Long-term outcome of subthalamic nucleus deep brain stimulation for Parkinson's disease using an MRI-guided and MRI-verified approach. J Neurol Neurosurg Psychiatry. 2014 Dec; 85(12):1419-25.

10 Erhardt JB, Fuhrer E, Gruschke OG, Leupold J, Wapler MC, Hennig J, et al. Should patients with brain implants undergo MRI? J Neural Eng. 2018 Aug;15(4):041002. 